\title{
ACCURACY OF MINISCREW INSERTION USING A CUSTOMIZED PRINTED THREE-DIMENSIONAL SURGICAL GUIDE (A COMPARATIVE SPLIT MOUTH STUDY)
}

\author{
Adel Rashid*, Hanem El Feky* and Noha Ossama AbdelAziz Issa**
}

\begin{abstract}
Aim of the study: This study was conducted to evaluate the accuracy of a new constructed 3D CBCT image-based surgical guide for miniscrew implantation .

Materials and Methods: Twenty five adult patients of were included in the current study (13 males and 12 females). Mandibular arch of every patient was randomly divided into : one experimental side (miniscrews were placed using surgical guide) and one comparison side (miniscrews were placed conventionally without surgical guide. For both sides (experimental and comparison side); Linear measurements were recorded on the software of coronal deviations, apical deviations and vertical deviations. Also, angular measurements were recorded in both mesiodistal and buccolingual dimensions for the actual plan and the real mini screws. At the end all deviations were calculated and statistical analysis was performed.
\end{abstract}

Results: A significantly higher level of accuracy (lower differences from the actual value) was achieved with the surgical guide technique $(\mathrm{p}<0.001)$ than hand drilling technique for all measurements .

Conclusions: Three Dimensional surgical guides provide a precise method for miniscrew placement into the dentoalveolar bone.

KEYWORDS: Miniscrew placement, Surgical guide,Accuracy

\section{INTRODUCTION}

Patient compliance is preferred to be minimal especially while providing maximal anchorage . For this reason, professionals started to use a wide array of anchorage devices, such as mini-plates, dental implants and miniscrews ${ }^{(1)}$.
In order to enhance orthodontic anchorage, these temporary anchorage devices are temporarily connected to the bone either by supporting the teeth of the reactive unit or by eliminating the need for the reactive unit altogether ${ }^{(2)}$. They could be transosteal, subperiosteal, or endosteal located and

\footnotetext{
* Lecturer of Orthodontics, Faculty of dentistry, Fayoum University, Fayoum, Egypt.

** Assistant Professor, Oral Radiology Department, Faculty of Oral and Dental Medicine, Cairo University .
} 
could be either mechanically (cortically stabilised) or biochemically (osseointegrated) fixed to bone. After usage, these devices are then disabled ${ }^{(3)}$.

The main advantages of the miniscrews are: their small size, low cost, streamlined insertion and removal surgical procedures, immediate load and finally providing adequate anchorage to enable orthodontic movements (2-5). Miniscrews can be inserted in a variety of maxillary and mandibular sites, notably in the interradicular spaces between adjacent teeth as well as in the palate and retromolar area ${ }^{(7-9)}$.

It is greatly important to determine the type and direction of the orthodontic tooth prior to miniscrew placement because insertion of the miniscrew at an unsuitable site could restrain the desired tooth movement. ${ }^{(6)}$. Also prior to insertion, both the site of placement and angulation of the miniscrew should be determined based on the patient's anatomical features . For example, if the amount of inter- radicular bone, its inclination or its proximity to the roots are not properly assessed, the risk of root perforation increases ${ }^{(10,11)}$.

There are two common strategies for miniscrew placement: self-tapping and self-drilling. In the selftapping procedure, a hole is drilled in the cortical bone before the miniscrew is mounted and a miniscrew is screwed through this hole with a hand driver (12). Clinicians should not apply too much pressure and irrigate the bone with coolants to minimize heat generation when drilling ${ }^{(13)}$. The miniscrew is inserted into the bone without drilling and screwed in with hand driver or engine driver in the self-drilling process ${ }^{(14,17)}$. Self-drilling screws have more boneto-metal contact than self-tapping screws and hence have better stability ${ }^{(15,16)}$.
Recent improvements in technology had led to development of the cone beam computed tomography (CBCT) specifically for the maxillofacial region. CBCT could provide sub-millimeter spatial resolution images with markedly shorter scanning times and has been reported to require somewhat lower radiation dosages than computed tomography (CT) imaging methods. CBCT allows personal computer-based two dimensional (2D) multi-planar reformatted (MPR) and secondary reconstruction of the data as well as volumetric three dimensional (3D) images that allows 1:1 image reality (11).

CBCT had also been linked with computerassisted design and computer-aided manufacturing (CAD-CAM) technology for dental implant placement ${ }^{(11)}$. However, it is still unknown whether CAD-CAM technique based surgical guidance is applicable to accurate and stable orthodontic miniscrew implantation or not ${ }^{(17)}$. Few types of 3D CBCT surgical guides had been recorded for miniscrew implantation ${ }^{(17,18)}$ and little ones regarding their accuracy ${ }^{(19)}$. Because of the above mentioned reasons, this research was conducted to evaluate the accuracy of a new constructed 3D CBCT image-based surgical guide for miniscrew implantation .

\section{MATERIALS AND METHODS}

\section{1- Sample size calculation}

A power analysis was designed to have an adequate power to apply a two-sided statistical test of the null hypothesis that there is no difference between both tested groups. By adopting an effect size $(\mathrm{d}=0.604)$ - calculated based on the results of Santis, et al., " an alpha $(\alpha)$ level of $0.05(5 \%)$, and a beta $(\beta)$ level of $0.20(20 \%)$ i.e. power $=80 \%$;

\footnotetext{
* Santis DD, Malchiodi L, Cucchi A, Cybulski A, Verlato G, Gelpi F and Nocini PF. "The Accuracy of ComputerAssisted Implant Surgery Performed Using Fully Guided Templates versus Pilot-Drill Guided Templates.” BioMed Research International; 2019 (april).
} 
the predicted sample size (n) was found to be a total of (24) cases. Sample size calculation was performed using $\mathrm{G}^{*}$ Power version 3.1.9.4 *.

\section{2- Patients}

Twenty five adult patients of ages range between 18-22 years (mean age 20.5 years) were included in the current study (13 males and 12 females). They were selected from the economic outpatients orthodontic clinics, Faculty of Dentistry, Fayoum University, Fayoum, Egypt . All the patients fulfilled the following criteria :

1. Full permanent dentitions

2. No congenital missing teeth

3. No skeletal discrepancies

4. Class I malocclusion with severe crowding in both maxillary and mandibular archs

5. Treatment plan for all patients was extraction of the four first premolars.

6. Maximum anchorage was indicated for all patients.

\section{3- METHODS}

Patients who met the eligibility criteria during recruitment were invited to participate in the current study which was carried out at Faculty of Dentistry, Fayoum University. Approval for the research proposal was obtained from Ethics Committee of the Faculty of Medicine, Fayoum University \{ Number (R156) in Session (79)\} . its aim were explained in details for all the patients individually and written consent terms were obtained from the patients and legal guardians.
At this time, one researcher opened an envelope that contained a card with the name of one mandibular side (right or left) which represented the experimental side, while the contralateral side was the comparison one. Therefore, mandibular arch of every patient was randomly divided into: one experimental side (miniscrews were placed using surgical guide) and one comparison side (miniscrews were placed conventionally without surgical guide). Placement of the miniscrews in both sides was performed by the same operator using the self -tapping technique.

\section{A- Orthodontic Procedures:}

Maxillary and mandibular fixed orthodontic appliances were used (American Orthodontics, 3524 Washington Avenue, Sheboygan, WI 53081) . After bands cementation and brackets bonding, extraction of the first premolars in the four quadrants. Miniscrews (American Orthodontics, 3524 Washington Avenue, Sheboygan,WI 53081) were used in the both archs for maximum anchorage (1.2 $\mathrm{mm}$ in diameter and $8 \mathrm{~mm}$ in length) and were placed between the first molars and the second premolars bilaterally $(5 \mathrm{~mm}$ from the gingival margins) .

In the mandibular arch, miniscrews were placed using the surgical guide (in the the experimental side), while in the contra lateral one were placed conventionally without the guid (free handing).

\section{Experimental side (surgical guide) :}

\section{1- Surgical guide construction:}

Cone beam CT $* *$ was performed to the patient and reconstructed as DICOM files. The stone model of the same patient was scanned using a laboratory optical scanner *** and exported as an STL format.

\footnotetext{
* Franz F. "G* Power 3: A flexible statistical power analysis program for the social, behavioral, and biomedical sciences." Behavior research methods 39.2 (2007): 175-191.

** Promax classic, Planmeca, Finland.

*** Open technologies, Brescia, Italy.
} 
On the implant planning software * registration of the optical scan of the cast to the cone beam CT was performed using fixed and clear landmarks such as incisal point angles, cusp tips, .. etc

Best fit was then performed by the software and the registration was checked by the operator. Implant planning was performed on the planning software for placement of the orthodontic mini screws placed in between teeth avoiding injury to any of the neighboring roots. The plan was exported to the cad software $* *$ for designing of the surgical guide. The surgical guide was then exported as an STL file. The guide was printed using a 3D printer $* * *$, followed by post curing (figure 1).

\section{2- Miniscrew insertion using surgical guide (Ex- perimental side):}

Miniscrews were placed using self-tapping technique. At the first, the surgical guide was seated in its proper position in the oral cavity which planned previously from the CBCT (figure 2). After that, a rotary surgical drill was used to make a hole

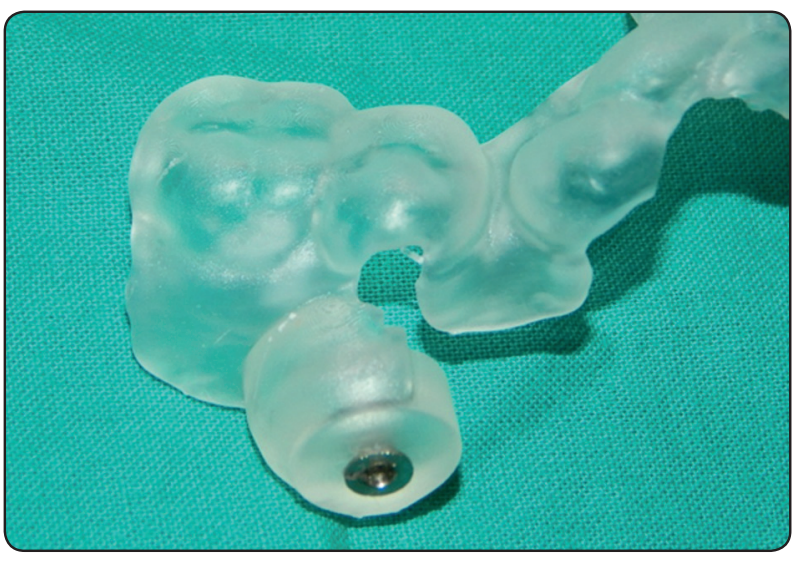

Fig. (1) Printed surgical guide in the cortical bone for about three millimeters depth through the guide (figure 3). During drilling, the operator applied minimal pressure and irrigated the bone with coolants to minimize the heat generation. Then the surgical guide was removed and the miniscrew was screwed through this hole using a hand driver (figure 4).

\section{Comparison side (free hand driving of the mini- screw)}

Insertion of the miniscrew was performed in an attempt to mimic the preplanned position of the implant on the software. Same steps were performed in the free hand group regarding the planning on the software without fabrication of a guide. Miniscrews were inserted clinically using self-tapping method as previously prescribed in the experimental side but without using the surgical guide . Before placing the miniscrew, a hole was drilled in the cortical bone using a rotary surgical drill for about three millimeters depth then the miniscrew is screwed through this hole with a hand driver ( figure 4).

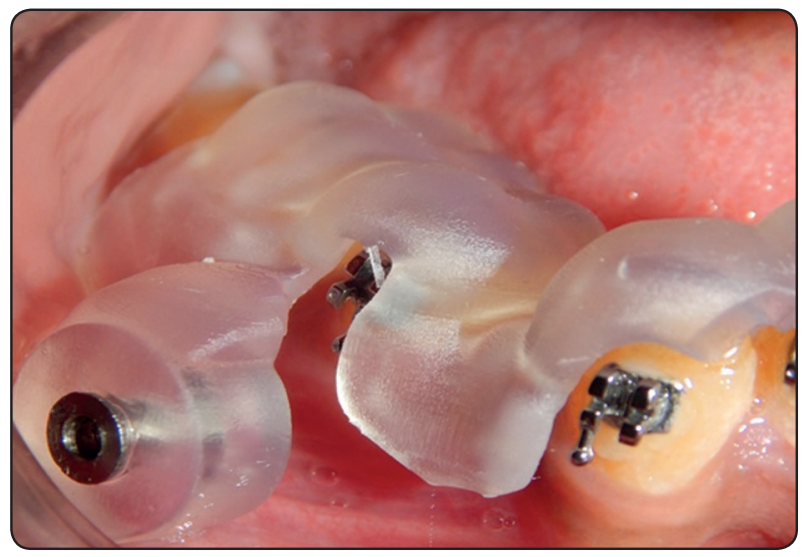

Fig. (2) The surgical guide seated in its planed position clinically.

\footnotetext{
* Diagnosys version 4.2, 3 Diemme, Como, Italy.

** Plastycad version 1.7, 3 Diemme, Como, Italy.

*** Form 2, Formlabs, USA.

***** Ondemand 3D, Cybermed, Korea.

(B) IBM Corporation, NY, USA.

(B) SPSS, Inc., an IBM Company.
} 


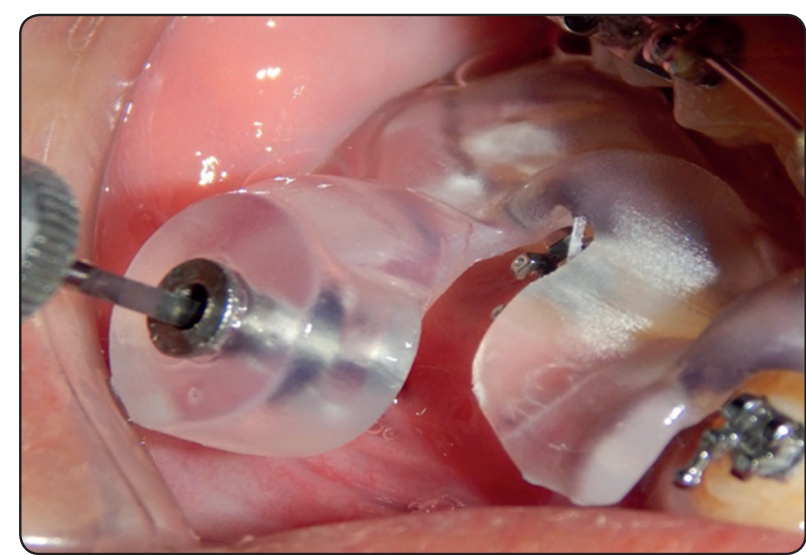

Fig. (3) Drilling a hole in the cortical bone using rotary surgical drill through the surgical guide.

\section{$B$ - Linear and angular digital measurements :}

Postoperative CBCT was performed for both groups followed by reslicing * of a new DICOM file based on DICOM registration of the postoperative CBCT to the preoperative CBCT ensuring same positions of both scans. thresholding was performed to the postoperative $\mathrm{CBCT}$ to separate the radiopaque mini screws from the bone. The miniscrews then were exported as STL. The mini screws exported in the previous step were imported to preoperative CBCT with the planning. A new virtual miniscrew was planned being at the exact same position of the imported STL mini screws.

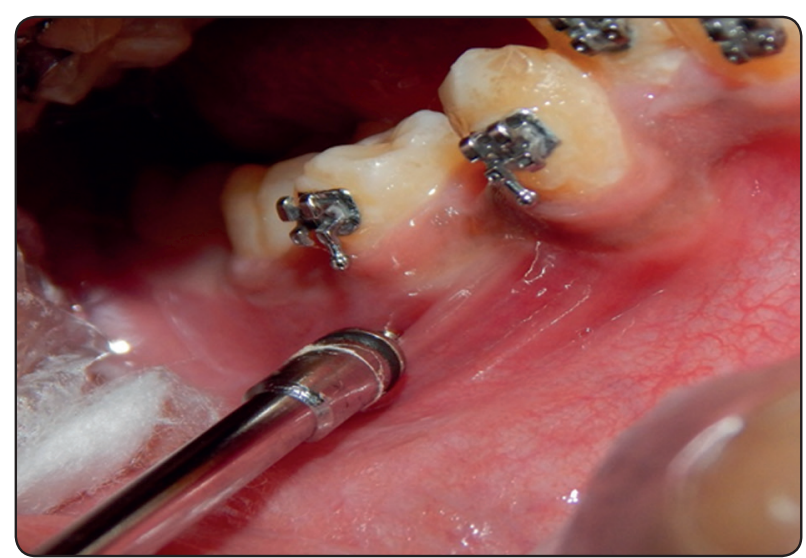

Fig. (4) Scuring the miniscrew in position with hand driver after hole driling .

For both sides (experimental and comparison side); Linear measurements were recorded on the software of coronal deviations, apical deviations and vertical deviations. Also, angular measurements were recorded in both mesiodistal and buccolingual dimensions for the actual plan and the real mini screws (figures 5\&6). Finally, all deviations were calculated.

All the above mentioned digital measurements were done blindly by the same researcher for all data obtained from experimental and comparison sides .

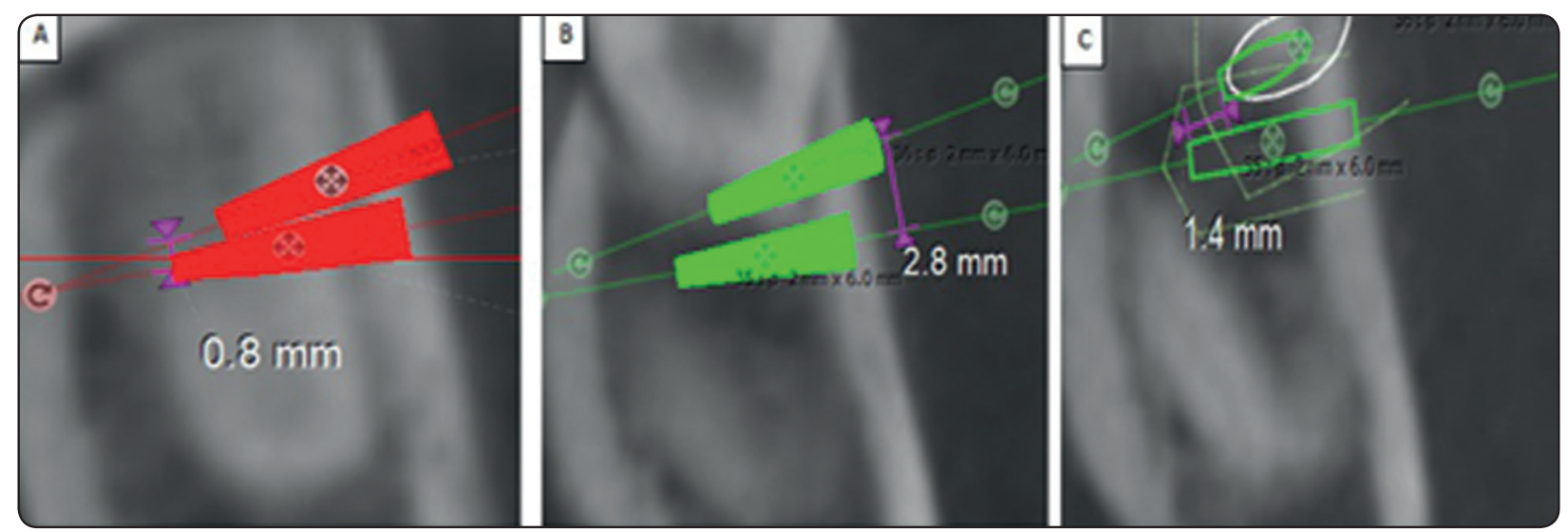

Fig. (5) The linear measurements in the free hand group (comparison side): (A) Apical deviation, (B) Coronal deviation and (C) Vertical deviation. 


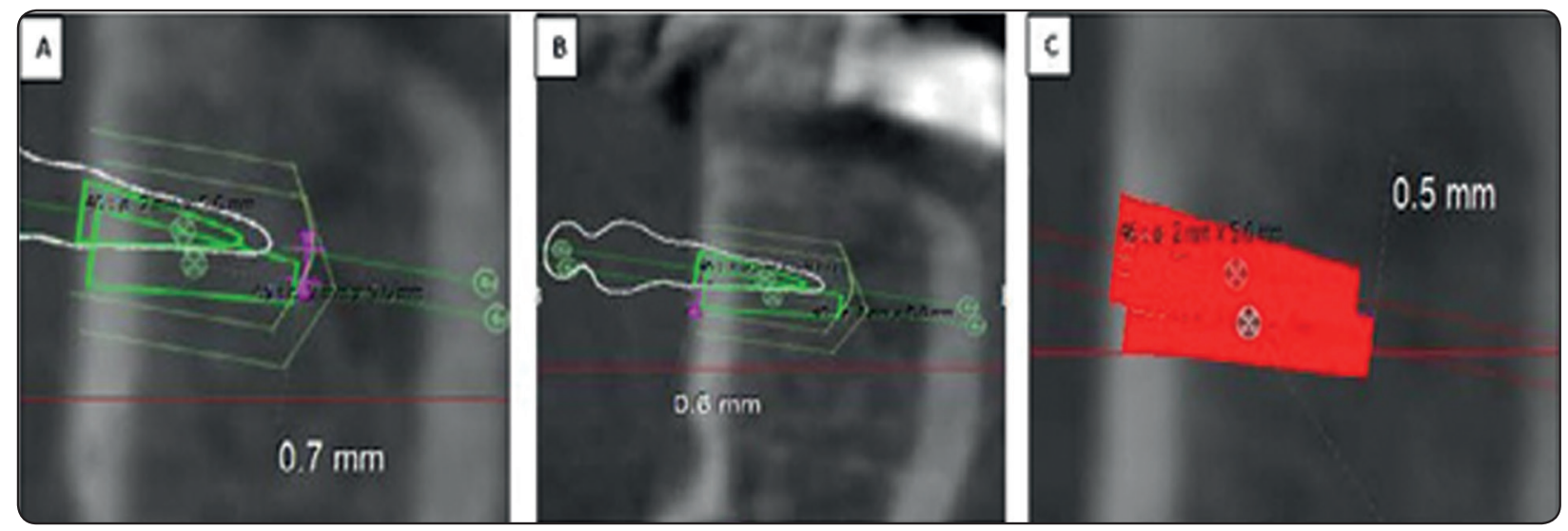

Fig. (6): The linear measurements in the surgical group (experimental side): (A) Apical deviation, (B) coronal deviation and (C) Vertical deviation.

\section{Statistical analysis:}

Numerical data were explored for normality by checking the data distribution, calculating the mean and median values and using Kolmogorov-Smirnov and Shapiro-Wilk tests. Data showed parametric distribution so it was represented by mean and standard deviation (SD) values. Paired t-test was used for intergroup comparison. Statistical analysis was performed with IBM ${ }^{\circledR}$ SPSS ${ }^{\circledR}$ Statistics Version 26 for Windows.

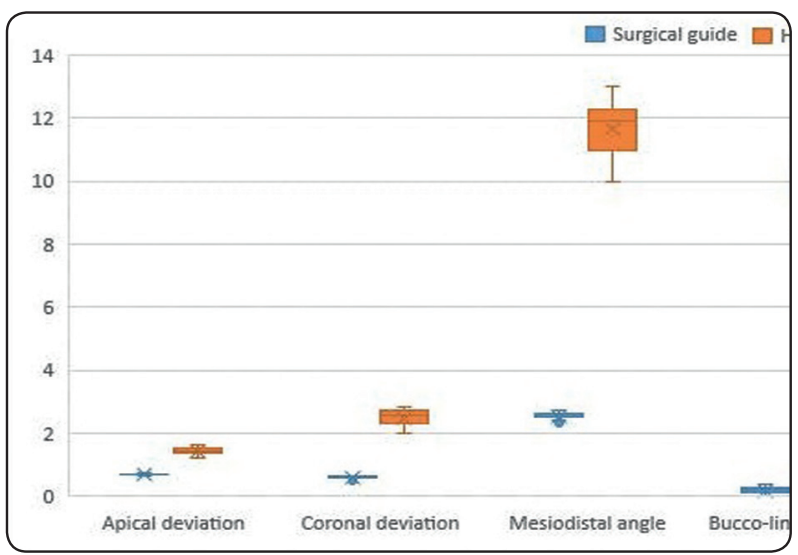

Fig. (7): Box plot chart showing the difference from actual values in different techniques and parameters

(®) IBM Corporation, NY, USA.

(®) SPSS, Inc., an IBM Company.

\section{RESULTS}

A significantly higher level of accuracy (lower differences from the actual value) was achieved with the surgical guide technique $(\mathrm{p}<0.001)$. Descriptive statistics for the difference from the actual value for different techniques and measured parameters were summarized in table (I) and figure (6). Average values for the difference in measurements were presented in figure (7). Summary of paired t-test results summarized in table (II) showed surgical guide technique to have a significantly higher accuracy than hand drilling technique for all measurements $(\mathrm{p}<0.001)$.

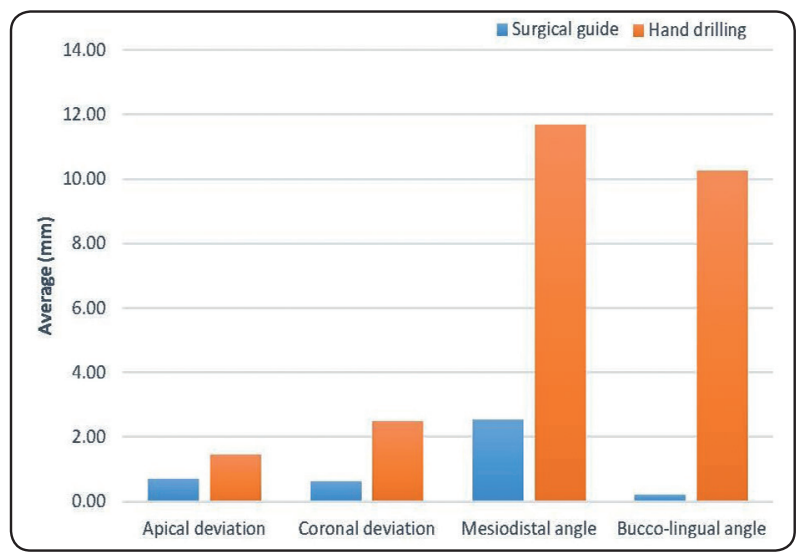

Fig. (8): Bar chart showing average difference from actual values in different techniques and parameters 
TABLE (I) Descriptive statistics for the difference from actual values in different teclmiques and parameters:

\begin{tabular}{|c|c|c|c|c|c|c|}
\hline Parameter & $\begin{array}{c}\text { Surgical } \\
\text { technique }\end{array}$ & Mean & $\begin{array}{c}\text { Std. } \\
\text { Deviation }\end{array}$ & Median & Min. & Max. \\
\hline \multirow{2}{*}{ Apical deviation } & Surgical guide & 0.69 & 0.02 & 0.70 & 0.65 & 0.75 \\
& Hand drilling & 1.44 & 0.10 & 1.44 & 1.22 & 1.60 \\
\hline \multirow{2}{*}{ Coronal deviation } & Surgical guide & 0.60 & 0.03 & 0.60 & 0.50 & 0.65 \\
& Hand drilling & 2.47 & 0.27 & 2.55 & 2.00 & 2.80 \\
\hline \multirow{2}{*}{ Mesiodistal angle } & Surgical guide & 2.53 & 0.10 & 2.50 & 2.30 & 2.70 \\
& Hand drilling & 11.67 & 0.75 & 11.90 & 10.00 & 13.00 \\
\hline \multirow{2}{*}{ Bucco-lingual angle } & Surgical guide & 11.18 & 0.09 & 0.20 & 0.00 & 0.36 \\
& Hand drilling & 10.25 & 0.91 & 10.55 & 8.50 & 11.60 \\
\hline
\end{tabular}

TABLE (II): Summary of paired $t$-test results:

\begin{tabular}{|c|c|c|c|c|c|}
\hline \multirow{2}{*}{ Parameter } & \multicolumn{2}{|c|}{$(\mathrm{Mean} \pm \mathrm{SD})$} & \multirow{2}{*}{$\begin{array}{l}\text { Mean difference } \\
\qquad(95 \% \mathrm{CI})\end{array}$} & \multirow{2}{*}{ t-value } & \multirow{2}{*}{ p-value } \\
\hline & Surgical guide & Hand drilling & & & \\
\hline Apical deviation & $0.69 \pm 0.02$ & $1.44 \pm 0.10$ & $-0.74(-11.78 ;-0.69)$ & -34.68 & $<0.001 *$ \\
\hline Coronal deviation & $0.60 \pm 0.03$ & $2.47 \pm 0.27$ & $-1.87(-1.97 ;-1.76)$ & -35.50 & $<0.001 *$ \\
\hline Mesiodistal angle & $2.53 \pm 0.10$ & $1 \mathrm{~L} 67 \pm 0.75$ & $-9.14(-9.46 ;-8.81)$ & -58.99 & $<0.001 *$ \\
\hline Bucco-lingual angle & $0.18 \pm 0.09$ & $10.25 \pm 0.91$ & $-10.34(-10.44 ;-9.71)$ & -56.56 & $<0.001 *$ \\
\hline
\end{tabular}

* significant $(p \leq 0.05)$

\section{DISCUSSION}

Ideal miniscrew placement (in the form of safety and stability) is affected by three critical factors: host factor (the morphology and quality of teeth and bone), the material factor (the conformation of the implants), and finally the operator factor (the placement techniques). Regarding the host factors, safe zones for miniscrew insertion had been reported. Many suggestions on miniscrew diameter and design, placement positions, and insertion angulations had been made due to the restricted width of the safe zone ${ }^{(14,15)}$.

One of the most common strategies to minimize the risks of root damage is to decrease the screw diameter and length, but implant fracture and low stability are frustrating when using thin miniscrews..
Improving the precision of the miniscrews placement techniques are therefore most important for the safety of implantation ${ }^{(15,16)}$.

Kuroda et al . ${ }^{(20)}$ concluded that root proximity was identified as a major risk factor of initial stability for miniscrews. They used two dimensional analysis (mainly periapical images) as their diagnostic aids which have inherent image distortions depending on X-ray tube angulation. Some CT studies have measured interradicular space for miniscrews to assess postsurgical position ${ }^{(21-23)}$

Liou et al ., ${ }^{(24)}$ recommended $2 \mathrm{~mm}$ of safety clearance space between OMI(Orthodontic Micro Implant) and the dental root; thus, a $1.5-\mathrm{mm}-$ diameter OMI would need $5.5 \mathrm{~mm}$ of interradicular septal width to ensure root safety and integrity which 
in turn making miniscrews placement impossible in most sites.

Contrary to these previous studies, Kim et al., ${ }^{(25)}$ showed that the surface-treated miniscrews could provide stationary anchorage through narrow interradicular space. Kim et al ., ${ }^{(26)}$ used CBCT to evaluate the root proximity and its effects on the stability of installed C-implants. They concluded that root proximity by itself was not a major risk factor for the miniscrew failure but could lead to loss of primary stability and subsequent loosening of the miniscrews.

Deguchi et al .. ${ }^{(2)}$ found that angulation of the placement of the miniscrews improved retention and recommended an angle of about $30 \mathrm{u}$ to the long tooth axis to improve the interdigitation of these miniscrews in the bone. At that oblique angle, the miniscrews would properly attain wider and longer distance through cortical bone and hence could achieve higher initial stability. However contrary to that, Byoun et al . ${ }^{(27)}$ concluded that the successful placement of the miniscrews is more closely related to the diameter and contact point of the miniimplant into the cortical bone surface rather than the insertion angle itself.

Three dimendional image programs are already being used to determine the accurate positioning and to make surgical guide for prosthetic implantation. Computed tomography scans are taken for evaluation of alveolar bone conditions, and the positions of implants are determined based on 3D digital images that are reconstructed from the computed tomography data ${ }^{(28)}$.

Reconstructing 3D digital images from computed tomography images alone is associated with several problems, such as distortion of the computed tomography image and artifacts caused by metal in the mouth or a beam-hardening effect. The use of digital model images comprising scanned images of plaster models combined with computed tomography images was reported to overcome this problems ${ }^{(29)}$.
In our study, the mesiodistal and buccolingual angular deviations were a mean of $2.53 \pm 0.10$ and $0.18 \pm 0.09$ respectively, and the linear apical and coronal distance deviations were means of $0.69 \pm 0.02$, $0.60 \pm 0.03$ respectively when the surgical used. While in the free hand drilling group the mesiodistal and buccolingual angular deviations were a mean of $11.67 \pm 0.75$ and $10.25 \pm 0.91$ respectively, and the linear apical and coronal distance deviations were means of $1.44 \pm 0.10,2.47 \pm 0.27$ respectively which proved that Miniscrews were placed more accurately when surgical guides were used than when a direct method was used.

The accuracy of surgical guides that were made with a $3 \mathrm{D}$ planning program based on CBCT images had also been reported elsewhere ${ }^{(28-38)}$. Liu et al .( ${ }^{(30)}$ reported that the angular deviation was $1.2 \pm 0.43_{\text {_ }}$, and the mesiodistal deviation was $0.42 \pm 0.13 \mathrm{~mm}$ at the apical area with surgical guides. Similarly, Morea et al . ${ }^{(31)}$ reported that the angular deviation was $1.76^{\circ}$, and the $3 \mathrm{D}$ linear distance deviations were $0.86 \mathrm{~mm}$ (coronal) and $0.87 \mathrm{~mm}$ (apex) using surgical guides.

Casseta et al ., ${ }^{(38)}$. stated in 2018 that the surgical guide enables controlled and precise palatal miniscrew placement in three dimentions. They found that the mean coronal and apical deviations were $1.38 \mathrm{~mm}$ (range: $3.48-0.15 \mathrm{~mm}$; standard deviation (SD): 0.65 ) and $1.73 \mathrm{~mm}$ (range: $5.41-0.10 \mathrm{~mm}$; SD: 1.03), respectively, while the mean angular deviation was $4.60^{\circ}$ (range: $15.23-0.54^{\circ}$; SD: 2.54 ).

Therefore, this study agreed with these previous studies ${ }^{(28-38)}$ and confirmed that the 3D surgical guide then provides a precise method for miniscrew placement into the dentoalveolar bone. The accurate insertion of miniscrews using the 3D surgical guide allows orthodontists to precisely transfer the radiographic information from preoperative planning to the surgical site, thus minimizing the risks of root injury. 


\section{CONCLUSIONS}

1- Three Dimensional surgical guides provide a precise method for miniscrew placement into the dentoalveolar bone.

2- Three Dimensional surgical guides allow orthodontist to minimize the risks of root injuries.

\section{REFERENCES}

1. Cousley R and Parberry DJ. Surgical stents for accurate miniscrew insertion. J Clin Orthod 1. 2006;40: 412-7.

2. Deguchi T, Nasu M, Murakami K, Yabuuchi T, Kamioka H and Takano-Yamamoto T. Quantitative evaluation of cortical bone thickness with computed tomographic scanning for orthodontic implants. Am J Orthod Dentofacial Orthop 2006; 129: 721.e7-721.e12.

3. Favero L, Brollo P and Bressan E. Orthodontic anchorage with specific fixtures: related study.analysis. Am J Orthod Dentofacial Orthop 2002; 121:84-94.

4. Kim H, Yun H, Park H, Kim D and Park Y. Soft-tissue and cortical-bone thickness at orthodontic implant sites. Am J Orthod Dentofacial Orthop 2006;130:177-82.

5. Kravitz D and Kusnoto B. Risks and complications of orthodontics miniscrews. Am J Orthod .Dentofacial Orthop 2007; 131:43-51.

6. Kuroda S, Sugawara Y, Degushi T, Kyung H and TakanoYamamoto T. Clinical use of miniscrew. implants as orthodontic anchorage: success rates and postoperative discomfort. Am J Orthod Dentofacial Orthop 2007;131:9-15.

7. Kyung S, Choi J and Park Y. Case report: miniscrew anchorage used to protract lower second molars into first molar extraction sites. J Clin Orthod 2003;10:575-9.

8. Lim W, Lee S, Wikesjö U and Chun Y. A descriptive tissue evaluation at maxillary interradicular sites: implications for orthodontic mini-screw placement. Clin Anat 2007; 20:760-5.

9. Liou EJ, Pai BCJ and Lin JCY. Do miniscrews remain stationary under orthodontic forces? Am.J Orthod Dentofacial Orthop 2004;1:42-7.

10. Motoyoshi M, Yoshida T, Ono A and Shimizu N. Effect of cortical bone thickness and implant placement torque on stability of orthodontic mini-screws. Int J Oral Maxillofac Implants 2007;22:779-84.
11. El-Beialy AR, Fayed MS, El-Bialy AM and Mostafa YA. Accuracy and reliability of cone-beam computed tomography measurements: Influence of head orientation. Am J Orthod Dentofacial Orthop. 2011 Aug; 140(2):157-65.

12. Yacker MJ, and Klein M. The effect of irrigation on osteotomy depth and bur diameter. International Journal of Oral Maxillofacial Implants 1996; 11(5): 634-638.

13. Matthews LS and Hirsch C. Temperatures measured in human cortical bone when drilling. Journal of Bone and Joint Surgery A1972; 54(2): 297-308.

14. Park HS. The usage of microimplants in orthodontics. In: R. Nanda and S. Kapila (ed.) Current therapy in orthodontics Mosby Inc. 2010; p291-300.

15. Kim JW, Ahn SJ and Chang YI. Histomorphometric and mechanical analyses of the drillfree screw as orthodontic anchorage. American Journal of Orthodontics and Dentofacial Orthopedics 2005;128(2): 190-194.

16. Kim YH and Choi JH. The study about retention of miniscrews used for intraoral anchorage. Journal of the Korean Dental Association 2001;39: 684-687.

17. Kim S, Choi Y, Hwang E, Chung K, Kook Y and Nelson G. Surgical positioning of orthodontic mini-implants with guides fabricated on models replicated with cone-beam computed tomography. Am J Orthod Dentofacial Orthop. 2007; 131(suppl 4):S82-S89.

18. Kim S, Kang J, Choi B and Nelson G. Clinical application of a stereolithographic surgical guide for simple positioning of orthodontic mini-implants. World J Orthod. 2008;9 :371-382.

19. Liu H, Liu DX,Wang G, Wang CL and Zhao Z. Accuracy of surgical positioning of orthodontic miniscrews with a computer-aided design and manufacturing template. Am J Orthod Dentofacial Orthop. 2010;137(6):728.e1-728.e10

20. Kuroda S, Yamada K, Deguchi T, Hashimoto T, Kyung $\mathrm{HM}$ and Takano-Yamamoto T. Root proximity is a major factor for screw failure in orthodontic anchorage. Am J Orthod Dentofacial Orthop. 2007;131:568-573.

21. Poggio PM, Incorvati C, Velo $\mathrm{S}$ and Carano A. "Safe zones": a guide for miniscrew positioning in the maxillary and mandibular arch. Angle Orthod. 2006;76: 191-197.

22. Herna'ndez L, Montoto G, Rodrı 'guez M, Galba'n L and Martı 'nez V. "Bone map" for a safe placement of miniscrews generated by computed tomography. Clin Oral Implants Res. 2008;19: 576-581. 
23. Park HS. An anatomical study using CT images for the implantation of micro-implants. Korean J Orthod. 2002; 32: 435-441.

24. Liou EJ, Pai BC and Lin JC. Do miniscrews remain stationary under orthodontic forces? Am J Orthod Dentofacial Orthop. 2004;126:42-47.

25. Kim SH, Cho JH, Chung KR, Kook YA and Nelson G. Removal torque values of surface-treated mini-implants after loading. Am J Orthod Dentofacial Orthop. 2008;134: 36-43.

26. Kim SH, Kang SM, Choi YS, Kook YA, Chung KR and Huang JC. Cone-beam computed tomography evaluation of mini-implants after placement: is root proximity a major risk factor for failure? Am J Orthod Dentofac Orthop. 2010;138:264-276.

27. Byoun NY, Nam EH, Yoon YA and Kim IK. Three-dimensional finite element analysis for stress distribution on the diameter of orthodontic mini-implants and insertion angle to the bone surface. Korean J Orthod. 2006;36:178-187.

28. Valente F, Schiroli G and Sbrenna A. Accuracy of computer-aided oral implant surgery: a clinical and radiographic study. Int J Oral Maxillofac Implants 2009;24: 234-42.

29. Bae MJ , Kim JY, Park JT, Cha JY, Kim HJ, Yu HS and Hwang CJ. Accuracy of miniscrew surgical guides assessed from cone-beam computed tomography and digital models. Am J Orthod Dentofacial Orthop.2013 Jun;143 (6):893-901.

30. Liu H, Liu DX, Wang G, Wang CL and Zhao Z. Accuracy of surgical positioning of orthodontic miniscrews with a computer-aided design and manufacturing template. Am J Orthod Dentofacial Orthop 2010; 137:728. e1-10; discussion 728-9.

31. Morea C, Hayek JE, Oleskovicz C, Dominguez GC and Chilvarquer I. Precise insertion of orthodontic miniscrews with a stereolithographic surgical guide based on cone beam computed tomography data: a pilot study. Int J Oral Maxillofac Implants. Jul-Aug 2011;26 (4):860-5.

32. Kim SH, ChoiYS, Hwang EW, Chung KR, Kook YA and Nelson G. Surgical positioning of orthodontic miniimplants with guides fabricated on models replicated with cone-beam computed tomography. Am J Orthod Dentofacial Orthop. 2007 Apr; 131(4 Suppl):S82-9.

33. Kim SH, Kang JM, Choi B and Nelson G. Clinical application of a stereolithographic surgical guide for simple positioning of orthodontic mini-implants. World J Orthod. Winter 2008; 9(4):371-82.

34. Yugo Suzuki E and Suzuki B. Accuracy of miniscrew implant placement with a 3-dimensional surgical guide. J Oral Maxillofac Surg.2008 Jun; 66(6):1245-52.

35. Miyazawa K, Kawaguchi M, Tabuchi M and Goto S. Accurate pre-surgical determination for self-drilling miniscrew implant placement using surgical guides and cone-beam computed tomography. Eur J Orthod 2010 Dec; 32(6): $735-40$.

36. Qiu L, Haruyama N, Suzuki S, Yamada D, Obayashi N, Toru Kurabayashi and Keiji Moriyama. Accuracy of orthodontic miniscrew implantation guided by stereolithographic surgical stent based on cone-beam CT-derived 3D images. Angle Orthod. 2012 Mar; 82(2):284-93.

37. Yu JJ, Kim GT, Choi YS, Hwang EH, Paek J, Kim SH and Huang JC. Accuracy of a cone beam computed tomography-guided surgical stent for orthodontic mini-implant placement. Angle Orthod.2012 Mar;82(2):275-83.

38. Cassetta M, Altieri F, Giorgio RD and Barbato E. Palatal orthodontic miniscrew insertion using a CAD-CAM surgical guide: description of a technique. Int J Oral Maxillofac Surg 2018 Sep; 47(9): 1195-1198. 